ARTICLE

DOI: $10.1038 / \mathrm{s} 41467-018-04189-6$

\title{
Giant energy density and high efficiency achieved in bismuth ferrite-based film capacitors via domain engineering
}

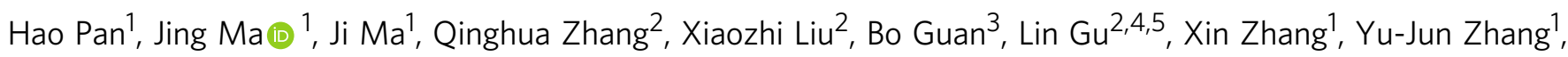
Liangliang Li (i) ${ }^{1}$, Yang Shen', Yuan-Hua Lin' ${ }^{1} \&$ Ce-Wen Nan ${ }^{1}$

Developing high-performance film dielectrics for capacitive energy storage has been a great challenge for modern electrical devices. Despite good results obtained in lead titanate-based dielectrics, lead-free alternatives are strongly desirable due to environmental concerns. Here we demonstrate that giant energy densities of $\sim 70 \mathrm{~J} \mathrm{~cm}^{-3}$, together with high efficiency as well as excellent cycling and thermal stability, can be achieved in lead-free bismuth ferritestrontium titanate solid-solution films through domain engineering. It is revealed that the incorporation of strontium titanate transforms the ferroelectric micro-domains of bismuth ferrite into highly-dynamic polar nano-regions, resulting in a ferroelectric to relaxorferroelectric transition with concurrently improved energy density and efficiency. Additionally, the introduction of strontium titanate greatly improves the electrical insulation and breakdown strength of the films by suppressing the formation of oxygen vacancies. This work opens up a feasible and propagable route, i.e., domain engineering, to systematically develop new lead-free dielectrics for energy storage.

\footnotetext{
${ }^{1}$ State Key Laboratory of New Ceramics and Fine Processing, School of Materials Science and Engineering, Tsinghua University, Beijing 100084, China. ${ }^{2}$ Beijing National Laboratory for Condensed Matter Physics, Institute of Physics, Chinese Academy of Sciences, Beijing 100190, China. ${ }^{3}$ Beijing National Laboratory for Molecular Science, Institute of Chemistry, Chinese Academy of Sciences, Beijing 100190, China. ${ }^{4}$ Collaborative Innovation Center of Quantum Matter, Beijing 100190, China. ${ }^{5}$ School of Physical Sciences, University of Chinese Academy of Sciences, Beijing 100049, China. Correspondence and requests for materials should be addressed to Y.-H.L. (email: linyh@tsinghua.edu.cn)
} 
D ielectric capacitors are the optimal option among currently available energy storage devices to offer the highest power density (on the order of Megawatt), highest operating voltage (several hundred to thousand volts) and longest work lifetime $^{1-3}$, which are ubiquitous and critical in modern electrical and electronic systems, especially in pulse power techniques including electrical weapon systems, hybrid electric vehicles and high-frequency inverters ${ }^{4,5}$. The key part in capacitors is the dielectric layer where electrostatic energy is stored in the form of electric displacement induced by an applied electric field. This unique energy storage mechanism leads to the intrinsic fast charging-discharging process and high power density. However, it also causes a relatively low energy density $\left(\sim 2 \mathrm{~J} \mathrm{~cm}^{-3}\right)$ in comparison with fuel cells or Li-ion batteries $\left(>20 \mathrm{~J} \mathrm{~cm}^{-3}\right)^{6,7}$. Therefore, developing dielectric materials with improved energy densities is imperative to enable the reduction of size, weight, and cost of cutting-edge electrical power systems.

The energy density $U_{\mathrm{e}}$ of dielectrics, which is determined by the applied electric field $E$ and the induced dielectric polarization $P$, can be mathematically expressed by

$$
U_{\mathrm{e}}=\int_{P_{\mathrm{r}}}^{P_{\mathrm{m}}} E \mathrm{~d} P
$$

where $P_{\mathrm{m}}$ and $P_{\mathrm{r}}$ are the maximum polarization and remnant polarization, respectively ${ }^{4}$ (Fig. 1a). Relaxor-ferroelectric (RFE) and antiferroelectric materials possess large $P_{\mathrm{m}}$ and small $P_{\mathrm{r}}$, both of which have been studied for capacitive energy storage ${ }^{5}$. Moreover, RFEs possess slim hysteresis loops that can be maintained at high electric fields, which results in high energy efficiency $\left(\eta\right.$, the ratio of $U_{\mathrm{e}}$ to the total stored energy density). Currently the mainstream RFE materials for energy storage are $\mathrm{PbTiO}_{3}$-based ceramics because of their high dielectric permittivity and strong polarization ${ }^{8}$. Yet bulk ceramics bear low breakdown strengths $\left(E_{\mathrm{b}}\right.$, the highest electric field a dielectric can sustain) due to the massive structural defects like pores and impurities, which hamper the realization of high energy density 9 . Recently, RFE thin films have been attracting increasing attention, where large $E_{\mathrm{b}}$ of $>1 \mathrm{MV} \mathrm{cm} \mathrm{cm}^{-1}$ is achievable owing to the improvement of film quality. Meanwhile, large $P_{\mathrm{m}}$ and relatively small $P_{\mathrm{r}}$ are maintained, giving rise to much elevated $U_{\mathrm{e}}$ values. For example, a $U_{\mathrm{e}}$ of $\sim 62 \mathrm{~J} \mathrm{~cm}^{-3}$ at a large $E_{\mathrm{b}}$ of $3.13 \mathrm{MV} \mathrm{cm}-1$ has been achieved in $\mathrm{Bi}\left(\mathrm{Ni}_{1 / 2} \mathrm{Ti}_{1 / 2}\right) \mathrm{O}_{3}-\mathrm{PbTiO}_{3} \mathrm{RFE}$ films ${ }^{10}$.

However, lead-containing materials pose strong threats to the environment and human health, which drives the intensive exploration of alternative lead-free materials. Some promising cases have been reported to date, among which the majority are modified $\mathrm{BaTiO}_{3}$ (BTO)-based RFE films. For instance, $U_{\mathrm{e}}$ values of $\sim 37 \mathrm{~J} \mathrm{~cm}^{-3}$ in $0.88 \mathrm{BaTiO}_{3}-0.12 \mathrm{Bi}(\mathrm{Mg}, \mathrm{Ti}) \mathrm{O}_{3}$ films $^{11}$ and $\sim 52 \mathrm{~J}$ $\mathrm{cm}^{-3}$ in $\mathrm{Ba}_{0.7} \mathrm{Ca}_{0.3} \mathrm{TiO}_{3}-\mathrm{BaZr}_{0.2} \mathrm{Ti}_{0.8} \mathrm{O}_{3}$ super-lattices ${ }^{12}$ have been reported recently. Nevertheless, there are still some drawbacks in those BTO-based RFEs that inhibit further improvements. First, the spontaneous polarization $P_{\mathrm{s}}$ of BTO is only $26 \mu \mathrm{C} \mathrm{cm}^{-2}$, much lower than that of $\mathrm{PbTiO}_{3}\left(\sim 80 \mu \mathrm{C} \mathrm{cm}^{-2}\right)^{13}$. Second, as BTO-based RFEs bears low Curie temperatures $T_{c}\left(<100^{\circ} \mathrm{C}\right)$, the deterioration of their ferroelectricity and energy storage performance can be evident at elevated temperatures ${ }^{14,15}$. To address these problems, we focus on a new lead-free system, namely, $\mathrm{BiFeO}_{3}$ (BFO), which has been acknowledged to be a promising alternative of lead-based dielectrics/ferroelectrics with a large $P_{\mathrm{s}}$ of $\sim 100 \mu \mathrm{C} \mathrm{cm}^{-2}$ and a high $T_{\mathrm{c}}$ of $830^{\circ} \mathrm{C}^{16}$. But pure $\mathrm{BFO}$ exhibits a large $P_{\mathrm{r}}$ due to strong ferroelectric (FE) hysteresis, restricting its usage for energy storage ${ }^{16}$. Modified BFO-based dielectrics such as BFO-BTO, BFO- $\left(\mathrm{Bi}_{1 / 2} \mathrm{Na}_{1 / 2}\right) \mathrm{TiO}_{3}$, and BFO-Pb $(\mathrm{Zr}, \mathrm{Ti}) \mathrm{O}_{3}$ have been reported to show RFE-like features ${ }^{17-19}$. Some preliminary work also found RFE properties with potentially good energy performance in $\mathrm{BFO}-\mathrm{SrTiO}_{3}$ systems ${ }^{20,21}$ However, the underlying mechanisms for the emergence of RFE features in BFO-based dielectrics and a feasible approach to design high-energy-density BFO-based RFEs remain undiscovered.

In this contribution, we present a domain engineering method to develop BFO-based RFEs with superior energy performance. A series of solid-solution $\left(\mathrm{BiFeO}_{3}\right)_{1-x}-\left(\mathrm{SrTiO}_{3}\right)_{x}$ (denoted as BFSTO, $0<x<1)$ films are designed and fabricated. It is revealed that the incorporation of $\mathrm{SrTiO}_{3}$ (STO) can transform the micrometer-scale FE domains of BFO into highly-dynamic polar nano-regions (PNRs), leading to a macroscopic FE-to-RFE transition (schematically shown in Fig. 1b). The favourable RFE state induced by domain engineering possesses simultaneously large $P_{\mathrm{m}}$ and suppressed $P_{\mathrm{r}}$, which, together with the enhanced breakdown strength with the STO incorporation, gives rise to giant energy densities with high energy efficiency of the BFSTO films.

\section{Results}

Microstructure. The BFSTO films were grown on the $0.7 \mathrm{wt} \%$ $\mathrm{Nb}$-doped STO (Nb:STO) single crystal substrates using a pulsed laser deposition (PLD) system. X-ray diffraction (XRD) patterns prove the highly preferential $c$-axis orientation of the films as revealed by the $(00 l)$ peaks without any trace of others (Fig. $2 \mathrm{a}$ and Supplementary Fig. 1). Phi scan of the $\{101\}$ planes (inset of Fig. 2a and Supplementary Fig. 1) further reveals that the films inherit a four-fold rotational symmetry from the substrate: $(001)_{\mathrm{BFSTO}} / /(001)_{\mathrm{Nb}} \mathrm{STO}$ (out of plane) and $[010]_{\mathrm{BFSTO}} / /$ $[010]_{\mathrm{Nb}: \mathrm{STO}}$ (in plane), indicating an excellent cubic-on-cubic
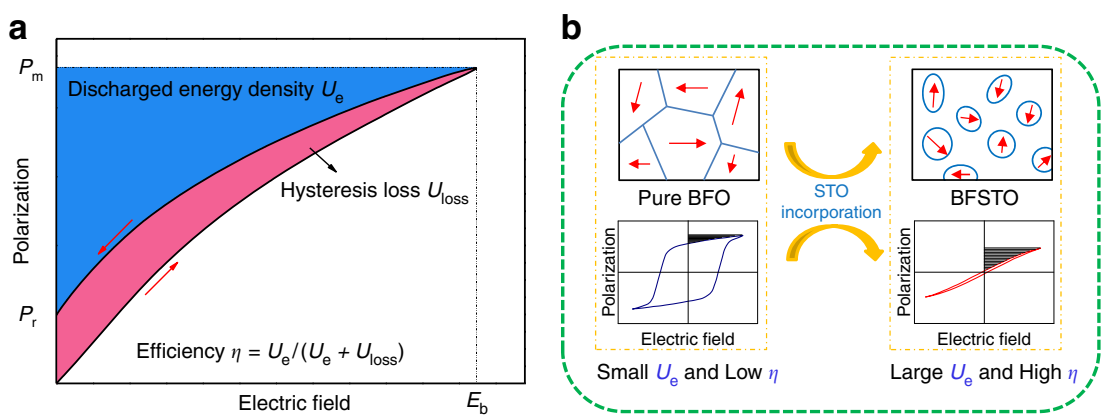

Fig. 1 Schematic illustrations. a A typical $P$-E loop of a dielectric and an illustration of (discharged) energy density $U_{\mathrm{e}}$, hysteresis loss $U_{\text {loss }}$ and efficiency $\eta$. The red arrows indicate the charging and discharging processes. $\mathbf{b}$ Schematic of domain evolution and FE-to-RFE transition induced by the incorporation of STO into BFO, leading to concurrently improved $U_{\mathrm{e}}$ and $\eta$. The blue outlines indicate the ferroelectric domains and the red arrows denote the spontaneous polarization directions 
a
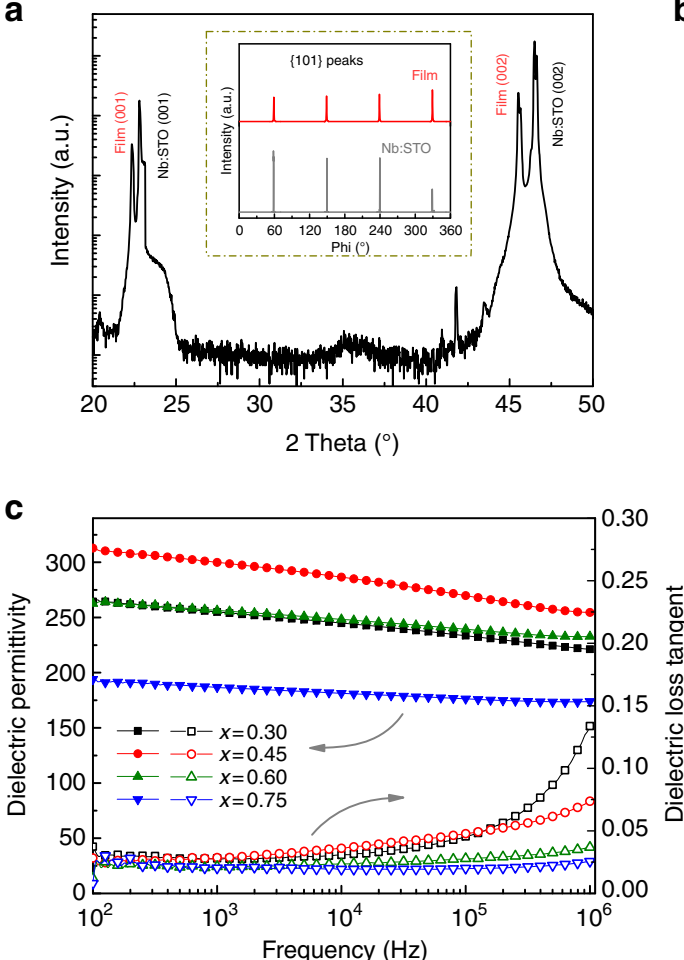

b

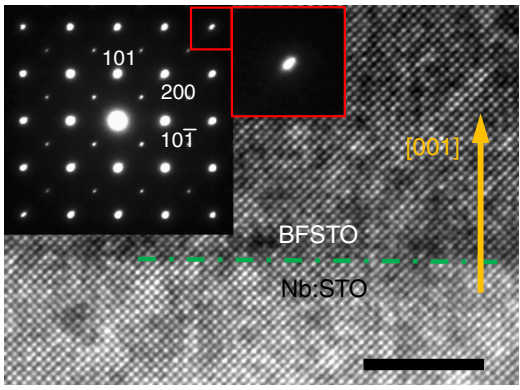

d

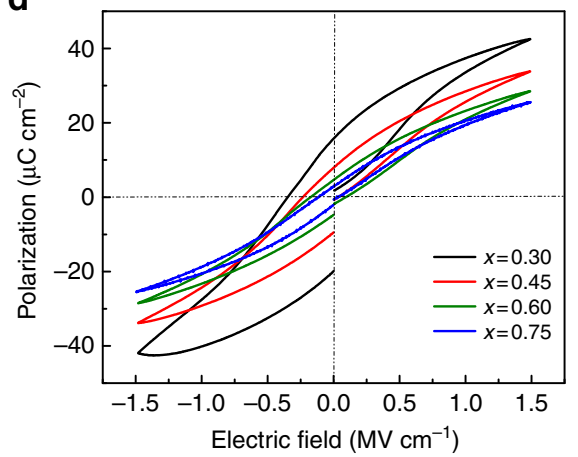

Fig. 2 Microstructure, dielectric, and ferroelectric properties. a XRD pattern of the BFSTO film with $x=0.45$; the insets shows the phi scans of its $\{101\}$ planes corresponding to the $\mathrm{Nb}$ :STO substrate. $\mathbf{b}$ A high-resolution TEM image of the interface of BFSTO film with $x=0.45$ and Nb:STO substrate (scale bar: $5 \mathrm{~nm}$ ); the inset is the SAED pattern at the interface zone. c Frequency-dependent dielectric permittivity and loss tangent and $\mathbf{d}$ bipolar $P$ - $E$ loops of the BFSTO films

epitaxy nature. The BFSTO films have a smooth surface with an average roughness of $\sim 1 \mathrm{~nm}$, as shown by an atomic force microscope image (Supplementary Fig. 2). A low-magnification high-angle angular dark-field (HAADF) image (Supplementary Fig. 3) displays clearly the sandwich structure consisting of the $\mathrm{Nb}$ :STO substrate, BFSTO film and Au top electrode. The BFSTO films are dense and crack-free, indicating good film quality due to the epitaxial growth. A direct evidence of the epitaxy is provided by a high-resolution transmission electron microscope (TEM) image (Fig. $2 \mathrm{~b})$ at the Nb:STO/BFSTO $(x=0.45)$ interface, which shows that both the film and matrix have single-crystal nature with a heteroepitaxial interface. The selected area electron diffraction (SAED) at the interface (inset of Fig. 2b) reveals that the BFSTO film shares the same type of diffraction pattern with the substrate. A small distortion of the diffraction spots shown in the red frame can be noticed because of the slight difference of lattice parameters between the BFSTO film and the Nb:STO substrate.

Dielectric and FE properties. Dielectric permittivity and loss tangent of the BFSTO thin films depending on frequency are displayed in Fig. 2c. It is interesting to note that the dielectric permittivity reaches the maximum of $\sim 300$ (at $1 \mathrm{kHz}$ ) in the film with $x=0.45$, which can be attributed to the rhombohedral-topseudo cubic phase boundary, in accordance with the findings in BFSTO bulk ceramics ${ }^{22}$. The permittivity declines gradually due to further increase of the paraelectric STO content, along with the loss tangent substantially reduced to 0.037 and 0.025 (at $1 \mathrm{MHz}$ ) for $x=0.60$ and 0.75 , respectively, which are at the same level with the best results reported in FE films, e.g. $\sim 0.05$ for PLZT film ${ }^{23}$ and $\sim 0.03$ for $\mathrm{Ba}(\mathrm{Zr}, \mathrm{Ti}) \mathrm{O} 3 \mathrm{film}^{24}$. The low loss tangent can prevent the self-heating and thermal runaway of dielectrics, which is crucial to the BFSTO films for capacitor applications ${ }^{25,26}$. Another remarkable feature of the BFSTO films is the tailoring of FE properties with the incorporation of STO. Bipolar $P-E$ hysteresis loops of the films measured with an electric field of $1.5 \mathrm{MV} \mathrm{cm}^{-1}$ are shown in Fig. $2 \mathrm{~d}$. One can see that the film with $x=0.30$ possesses the largest $P_{\mathrm{m}}$ and $P_{\mathrm{r}}$ with remarkable hysteresis, indicating typical FE properties. Notably, films with increasing $x$ exhibit much narrowed $P-E$ loops with substantial reduction of $P_{\mathrm{r}}$, which represents a crossover towards RFE feature. The temperature-dependent dielectric spectroscopies of the BFSTO films exhibit broadened dielectric peaks and frequency dispersion (Supplementary Fig. 4), further evidencing the RFE characteristics $^{14}$.

Energy storage performance. The discharged energy densities of the BFSTO films are determined from their unipolar $P-E$ loops (Supplementary Fig. 5) and plotted in Fig. 3a. It is most striking that the film with $x=0.60$ exhibits a giant $U_{\mathrm{e}}$ of $70.3 \mathrm{~J} \mathrm{~cm}^{-3}$ at $3.85 \mathrm{MV} \mathrm{cm}^{-1}$; the film with $x=0.75$ also achieves a $U_{\mathrm{e}}$ of $70.0 \mathrm{~J} \mathrm{~cm}^{-3}$ at $4.46 \mathrm{MV} \mathrm{cm}^{-1}$. Although the films with $x=0.45$ or 0.30 possess higher $P_{\mathrm{m}}$ values, they also bear larger $P_{\mathrm{r}}$ (Supplementary Fig. 5), probably because of the larger FE hysteresis and higher leakage current at high electric fields ${ }^{27}$. As a result, their energy densities are substantially limited. For example, the film with $x=0.30$ shows a maximum $U_{\mathrm{e}}$ of only $21.8 \mathrm{~J} \mathrm{~cm}^{-3}$ at 2.75 $\mathrm{MV} \mathrm{cm}-1$, which is even smaller than that $\left(36.1 \mathrm{~J} \mathrm{~cm}^{-3}\right)$ of $x=$ 0.75 at the same electric field. The large $P_{\mathrm{r}}$ also causes remarkable decline of energy efficiency $\eta$ of $x=0.30$ and 0.45 , as shown in Fig. 3b. In sharp contrast, high $\eta$ values of $70 \%$ at $3.85 \mathrm{MV} \mathrm{cm}^{-1}$ and $68 \%$ at $4.46 \mathrm{MV} \mathrm{cm}^{-1}$ are maintained for $x=0.60$ and 0.75 , respectively. Note that high efficiency is also crucial for dielectrics because it means less waste heat, better reliability and longer lifetime of capacitors in practical applications. The energy densities and efficiency of the BFSTO films are compared with those of representative lead-based and lead-free material systems 
a

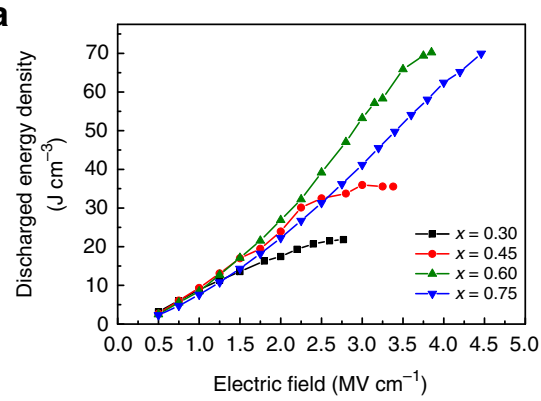

b

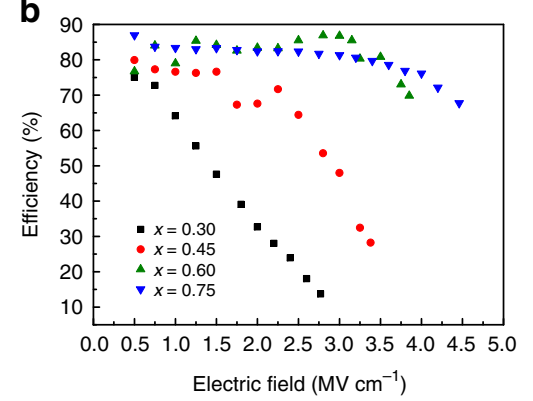

C
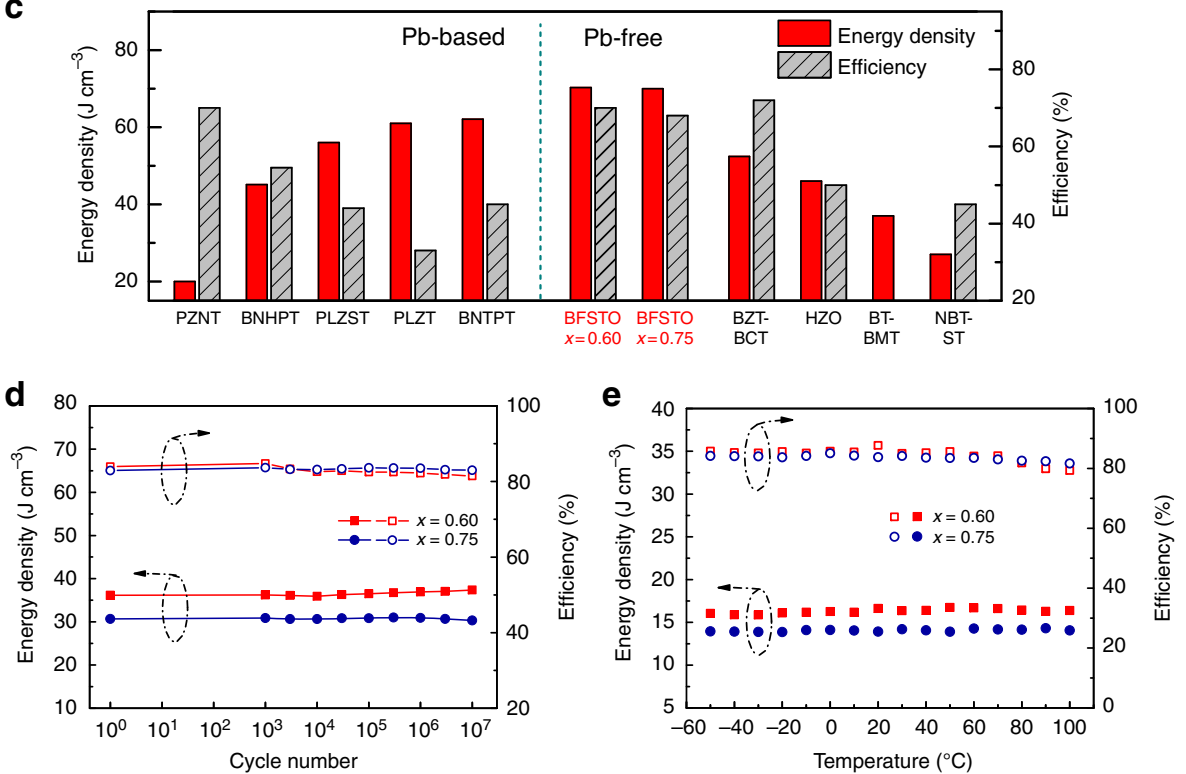

Fig. 3 Energy storage performance. a Discharged energy density and $\mathbf{b}$ efficiency of the BFSTO films as a function of the applied electric field. $\mathbf{c}$ Comparisons of energy density and efficiency between BFSTO and representative dielectric systems ${ }^{10-12,28-33}$, showing that the BFSTO films possess the most outstanding energy storage performance. $\mathbf{d}$ Energy density and efficiency for $x=0.60$ and 0.75 at an electric field of $2.5 \mathrm{MV} \mathrm{cm}^{-1}$ over $1 \times 10^{7}$ charging-discharging cycles. e Temperature-dependent energy storage performance for $x=0.60$ and 0.75 at an electric field of $1.5 \mathrm{MV}_{\mathrm{cm}}^{-1}$

reported previously, as displayed in Fig. $3 \mathrm{c}^{10-12,28-33}$. The results evidently show that the energy densities of the BFSTO films are superior to those of other reported lead-free systems (35\% over the best BTO-based systems ${ }^{12}$ ) and rival the lead-based materials. Additionally, the concomitantly achieved high efficiency makes the BFSTO films more attractive for energy storage applications.

Long-term working stability is another significant requirement for dielectrics. Fast charging-discharging cycling test has been conducted with a triangle electric field of $10 \mathrm{kHz}, 2.5 \mathrm{MV} \mathrm{cm}^{-1}$. It can be clearly seen in Fig. $3 \mathrm{~d}$ that the films with $x=0.60$ and 0.75 exhibit little degradation of either $U_{\mathrm{e}}$ or $\eta$ over $1 \times 10^{7}$ cycles. The excellent stability can be ascribed to (1) the robust mechanical strength of the films that prevents mechanical damage from electrostatic force and (2) the nearly defect-free microstructure and highly-dynamic RFE feature that inhibit domain wall pinning during the repeated polarization switching ${ }^{34}$. Thermal stability of the energy performance of BFSTO films was also investigated. The results (Fig. 3e) demonstrate that both the films with $x=0.60$ and 0.75 possess highly stabilized energy density and efficiency in a wide temperature range of -50 to $100^{\circ} \mathrm{C}$, which meets well with the requirement for applications in harsh environment such as aerospace and hybrid electric vehicles. The films with $x=0.30$ and 0.45 , on the contrary, show deterioration of energy performance at slightly elevated temperatures (Supplementary Fig. 6). This should be pinned on the increase of electrical conduction loss and similar phenomena have been reported in polymer and ceramic dielectrics ${ }^{6,35}$. The conduction loss and FE loss of the BFSTO films at various temperatures are decoupled based on the method by Khanchaitit et al. ${ }^{36}$, as shown in Supplementary Fig. 7 and Supplementary Note 1. It is found that the suppression of conduction loss for $x=0.60$ and 0.75 is the main contributor to the thermal stabilization of energy performance.

FE-to-RFE transformation and domain evolution. The outstanding energy performance of the BFSTO films should be ascribed to their favourable RFE properties. On the one hand, due to the existence of highly-polarizable $\mathrm{BFO}$ component, large $P_{\mathrm{m}}$ values are maintained, e.g., $\sim 59.2 \mu \mathrm{C} \mathrm{cm}^{-2}$ for $x=0.60$, representing at least $50 \%$ improvement over those of BTO-based RFEs $\left(<40 \mu \mathrm{C} \mathrm{cm}^{-2}\right)^{11,12}$ and thus guarantees an increased $U_{\mathrm{e}}$. On the other hand, the FE-to-RFE transformation markedly suppresses the $P_{\mathrm{r}}$ values, e.g., $\sim 11.1 \mu \mathrm{C} \mathrm{cm}^{-2}$ for $x=0.60$, hence inducing the minimized hysteresis loss and high $\eta$. In order to gain more insights into the FE-to-RFE transformation, first order reversal curve (FORC) distributions of the BFSTO films are investigated, which is a sensitive method to give reasonable descriptions of hysteresis behaviors ${ }^{37}$. The FORC method is based on the Preisach mode ${ }^{38,39}$ assuming that a hysteresis loop consists of a set of rectangular loops (termed as "hysterons") characterized by the positive-switching coercive field $\alpha$ and the negative-switching coercive field $\beta(\beta \leq \alpha$, schematically shown in Supplementary Fig. 8). The hysterons have a distribution in regard to $\alpha$ and $\beta$, 


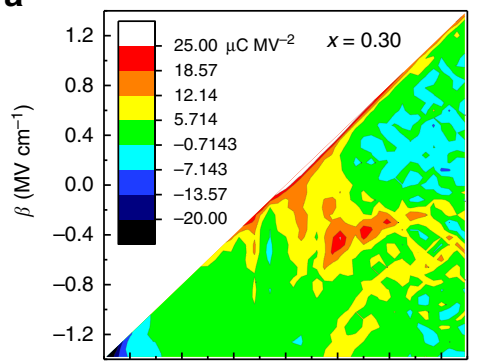

b

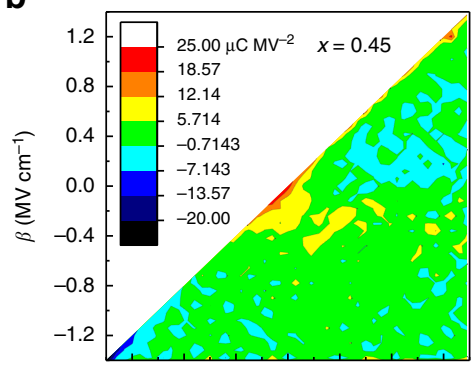

C

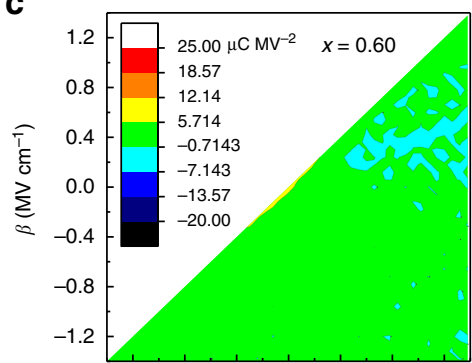

d
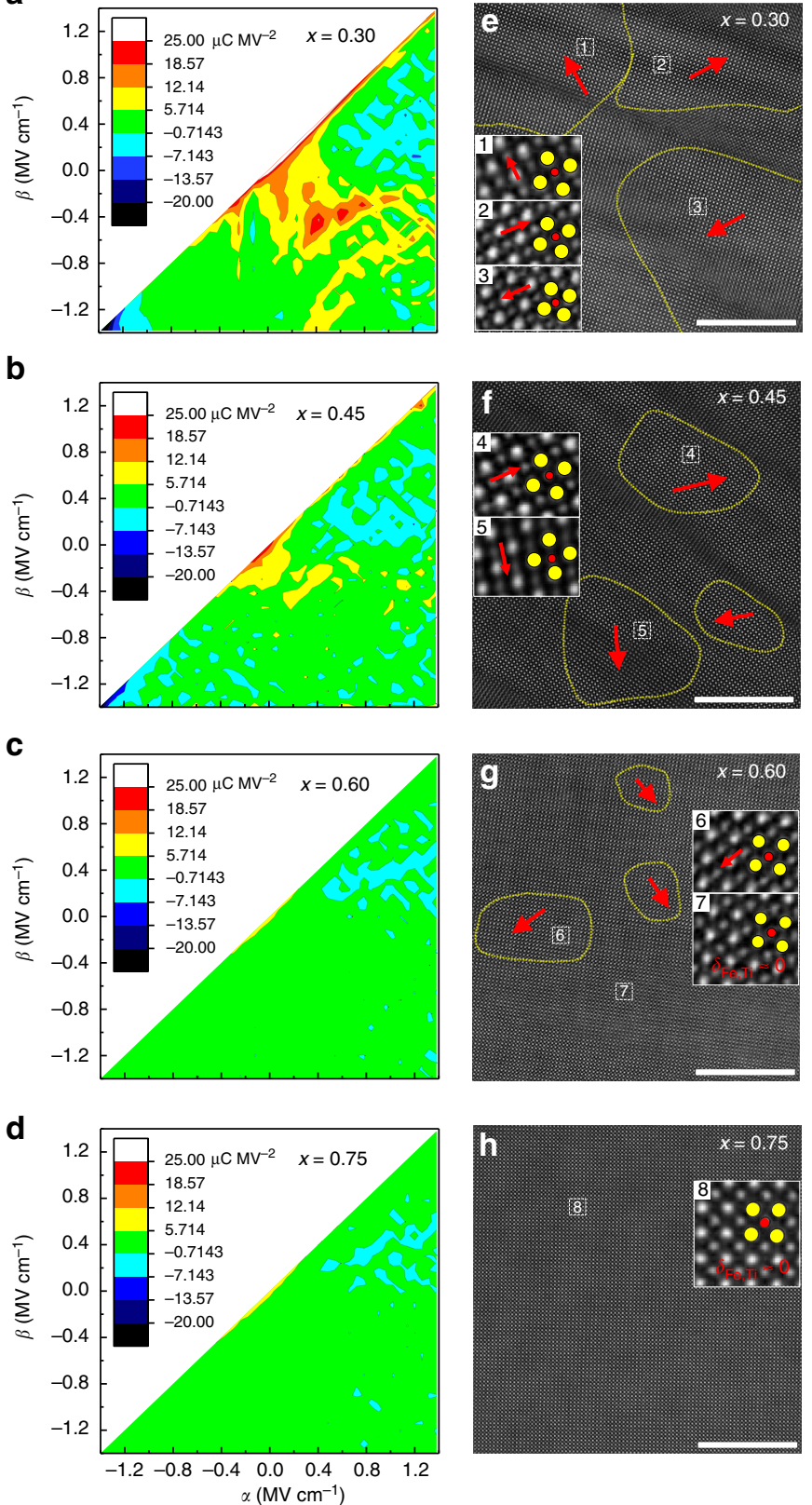

Fig. 4 Evolution of FORC distribution and domain configuration. a-d FORC distribution $p(\alpha, \beta)$ and $\mathbf{e - h}$ HAADF-STEM images of the atomic-scale ferroelectric domain structure of the BFSTO films (scale bars: $10 \mathrm{~nm}$ ). The yellow dashed lines in $\mathbf{e}-\mathbf{h}$ mark the domains with spontaneous polarization and the red arrows denote the $\mathrm{Fe} / \mathrm{Ti}$ ion displacement $\left(\delta_{\mathrm{Fe} / \mathrm{Ti}}\right)$ directions. The insets of $\mathbf{e}-\mathbf{h}$ are magnified images of selected areas to show the $\delta_{\mathrm{Fe} / \mathrm{Ti}}$, where the yellow and red circles denote the $\mathrm{Bi} / \mathrm{Sr}$ and $\mathrm{Fe} / \mathrm{Ti}$ ion columns, respectively

which is the FORC distribution $p(\alpha, \beta)^{39,40}$. In this work, the $p(\alpha$, $\beta$ ) diagrams of the films were derived from a series of FORC loop measurements (Supplementary Figs. 8 and 9). Based on the evolution of the diagrams shown in Figs $4 \mathrm{a}-\mathrm{d}$, the crossover from FE towards RFE state with increasing STO content has been illustrated. The diagram of the film with $x=0.30$ (Fig. 4a) exhibits a high-intensity distribution zone located near the origin point, which represents the switching of FE domains at lower electric fields, and is responsible for the large total polarization of the film ${ }^{41}$. The $p(\alpha, \beta)$ intensity at high-electric-field zones, however, is substantially suppressed due to the saturation of domain switching. This inhomogeneous distribution reflects the typical FE characteristics with strong polarization nonlinearity ${ }^{38}$. As $x$ increases to 0.45 (Fig. $4 \mathrm{~b}$ ), the high-intensity zones significantly shrink toward the origin point, indicating smaller coercive fields and decreased nonlinearity. This suggests a shift toward RFE state, since RFE is characterized with small energy barrier for switching and ultralow coercivity. When $x$ further increases to 0.60 and 0.75 (Fig. $4 c, d$ ), $p(\alpha, \beta)$ becomes more evenly distributed in the whole electric field range, indicating further weakened FE nonlinearity and dispersed polarization contribution in the RFE state ${ }^{40}$.

From a microscopic perspective, the RFE properties are proposed to be linked with the emergence of PNRs, which have been observed in both lead-based and lead-free RFE materials ${ }^{8,42}$. Theoretical simulations have offered clear insights into the existence of PNRs in the $\mathrm{Ba}(\mathrm{Zr}, \mathrm{Ti}) \mathrm{O}_{3}$ system $^{43}$, and the evolution of PNRs under electric fields that causes RFE properties ${ }^{44}$. Accordingly, it is proposed that the incorporated STO into BFO ( $\mathrm{Sr}$ into $\mathrm{Bi}$ sites and $\mathrm{Ti}$ into $\mathrm{Fe}$ sites) induces compositional and chemical disorder in the BFSTO films, breaking the long-range $\mathrm{FE}$ order of BFO and transforming the FE domains into PNRs, thus resulting in a RFE state ${ }^{45}$. The FE domains of pure BFO films can be observed with the piezoelectric force microscope (PFM) technique (Supplementary Fig. 10), which are of several hundred nanometer to several micrometer and strongly coupled with each other. To validate the emergence of PNRs in the RFE BFSTO films, atomic-scale characterizations based on the scanning transmission electron microscopy (STEM) is employed $^{46}$. In the HAADF $Z$-contrast STEM images shown in Figs $4 \mathrm{e}-\mathrm{h}$, the $\mathrm{A}$-site ion columns $\left(\mathrm{Bi}^{3+}\right.$ and $\left.\mathrm{Sr}^{2+}\right)$ appear as the brighter dots while $\mathrm{B}$-site ion columns $\left(\mathrm{Fe}^{3+}\right.$ and $\left.\mathrm{Ti}^{4+}\right)$ show weaker contrast. The displacements of $\mathrm{Fe} / \mathrm{Ti}$ ions relative to the lattice center can be directly observed in the images (see the magnified zones in the insets). The spontaneously polarized zones, i.e., the PNRs are schematically marked with yellow dashed lines, and the red arrows show the directions of $\mathrm{Fe} / \mathrm{Ti}$ ion displacement vector $\delta_{\mathrm{Fe} / \mathrm{Ti}}$. One can first note the decrease tendency of the area of long-range polarization order with increased STO content, which means a weakened ferroelectricity and is in accordance with Fig. 2d. As for the domain configuration, the results demonstrate an apparent picture that large-sized domains transform gradually into nano-scale PNRs as the STO content increases. The distance between neighboring PNRs also increases, implying weakened inter-coupling and enhanced domain switching mobility ${ }^{40,47}$. It should be pointed out that the domain structure of the film with $x=0.75$ is hard to distinguish, probably because the PNR sizes become so small that the ion displacements are submerged by the average effect of ion column projection. Besides the STEM images, the domain evolution in the BFSTO films can be also verified by the characterization of domain switching with PFM. As shown in Supplementary Fig. 11, pure BFO film exhibits stable retention of polarization at least $10 \mathrm{~h}$ after being poled with a voltage of $20 \mathrm{~V}$. In stark contrast, in the film with $x=0.60$, the polarized domains switch back mostly in $30 \mathrm{~min}$ after the applied voltage is removed. This phenomenon verifies the much enhanced domain mobility in the BFSTO films, indicating the occurrence of domain evolution $^{48}$.

Breakdown strength. Improved breakdown strength is another important factor for the superior energy performance of the BFSTO films since it allows a larger applied electric field and full polarization. The characteristic breakdown field $E_{\mathrm{b}}$ of the films was obtained using a two-parameter Weibull distribution fitting ${ }^{7}$, as plotted in Fig. 5a. Notably, the BFSTO films exhibit much 
a

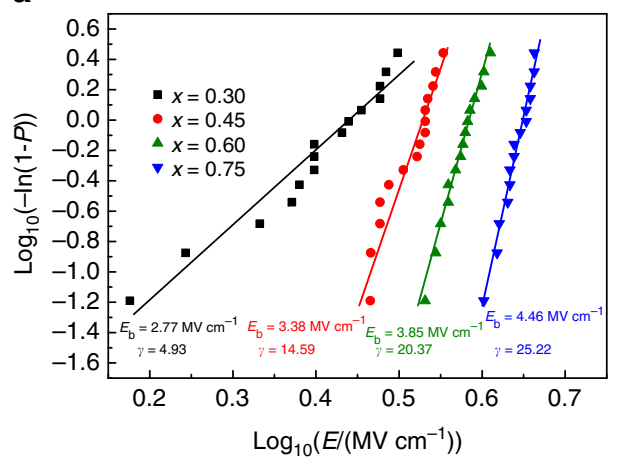

C

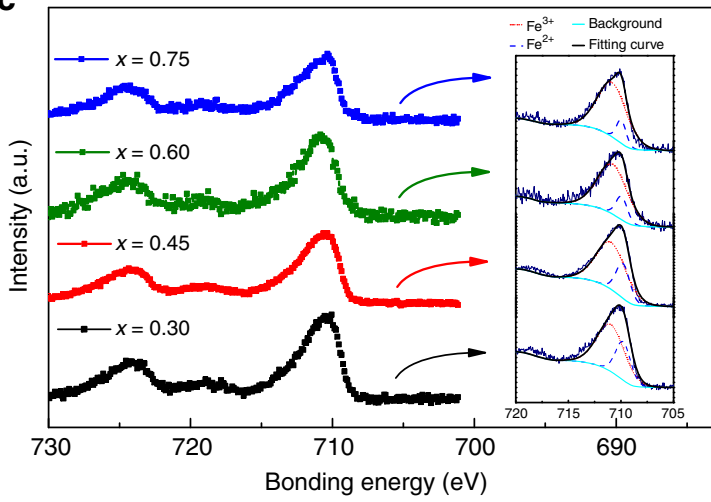

b

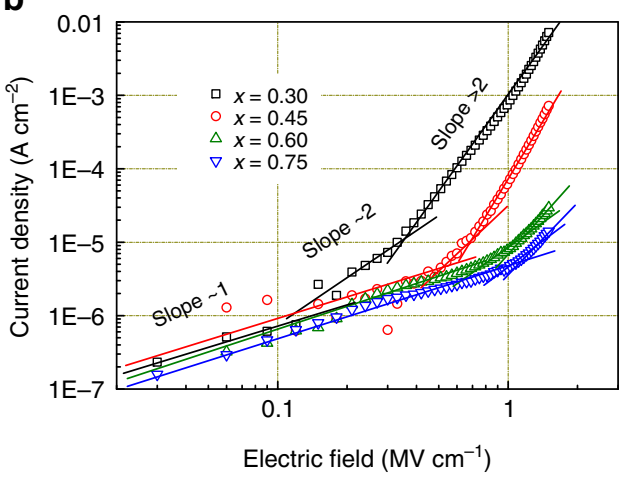

d

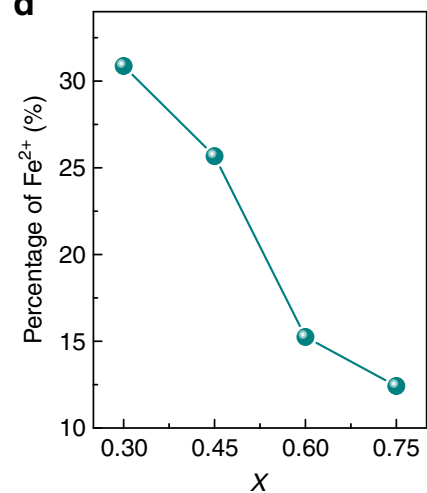

Fig. 5 Breakdown strength and electric conduction. a Two-parameter Weibull Distribution analysis of dielectric breakdown strengths, b room-temperature leakage current densities as a function of biased electric field, $\mathbf{c}$ XPS fitting for Fe valences and $\mathbf{d}$ percentage of Fe ${ }^{2+}$ out of all Fe ions in the BFSTO films

improved breakdown strengths that are positively related to the STO content. The $E_{\mathrm{b}}$ values of the films with $x=0.60$ and 0.75 reach $3.85 \mathrm{MV} \mathrm{cm}^{-1}$ and $4.46 \mathrm{MVcm}^{-1}$, respectively, which represent a nearly twofold enhancement over that of pure BFO film $\left(\sim 1.5 \mathrm{MV} \mathrm{cm}^{-1},\right)^{49}$ and are among the highest values in ceramic films ${ }^{12,32,33}$. Moreover, the Weibull modulus $\gamma$ that evaluates the scatter of breakdown field data also increases with the STO content. For example, $y=4.93$ and 25.22 for $x=0.30$ and 0.75 , respectively, suggesting a narrowed distribution of $E_{\mathrm{b}}$ values, namely, an improvement of dielectric reliability ${ }^{36}$. The pronounced enhancement of breakdown performance is reasonably ascribed to the improved electric insulation in the BFSTO films ${ }^{5}$. Figure $5 \mathrm{~b}$ shows that the steady-state leakage current $J$ of the BFSTO films is reduced by several orders of magnitude with increased STO content, e.g., from $7.2 \times 10^{-3} \mathrm{~A} \mathrm{~cm}^{-2}$ for $x=0.30$ to $1.4 \times 10^{-5} \mathrm{~A} \mathrm{~cm}^{-2}$ for $x=0.75$ at a bias field of $1.5 \mathrm{MV} \mathrm{cm}^{-1}$. With a closer look at the $J-E$ curves, one can notice that all samples follow Ohm's law at low electric fields $\left(\log _{10} J \sim S \log _{10} E\right.$, where the slope $S \approx 1$ ). As the electric field exceeds a critical value $E_{0}$, new conduction mechanisms with various sources of charge carriers become dominant, which in our case are the space charge limited conduction $\left(\log _{10} J \sim S \log _{10} E\right.$, where $\left.S \approx 2\right)$ at medium fields and the Schottky/Poole-Frenkel emission at high fields ${ }^{50,51}$. The specific $E_{0}$ values are $0.13,0.52,0.81$, and $1.05 \mathrm{MV} \mathrm{cm}^{-1}$ for $x=0.30,0.45,0.60$, and 0.75 , respectively, which indicates that the ability of inhibiting new charge carriers is enhanced by incorporating STO. As a matter of fact, the main charge carriers in BFO-based materials are acknowledged to be oxygen vacancies induced by the chemical valence fluctuation of $\mathrm{Fe}$ ions ${ }^{49}$. The corresponding reaction is written as

$$
2 \mathrm{Fe}_{\mathrm{Fe}}+\mathrm{O}_{\mathrm{O}} \rightarrow 2 \mathrm{Fe}_{\mathrm{Fe}}{ }^{\prime}+\mathrm{V}_{\mathrm{O}} \cdot \frac{1}{2} \mathrm{O}_{2}
$$

Hence a straightforward way to reduce the leakage current is to inhibit the $\mathrm{Fe}^{3+}$-to- $\mathrm{Fe}^{2+}$ transition, which has been proved effective in BFO-based ceramics with partially substitution of analogous ions (such as $\mathrm{Ti}, \mathrm{Mn}, \mathrm{Co}$, and $\mathrm{Al}$ etc.) at $\mathrm{Fe}$ sites $^{49,52}$ or BFO-ABO ${\text { (such as } \mathrm{PbTiO}_{3} \text { and BTO) solid solutions }}^{53,54}$. In the BFSTO system, we reveal that the $\mathrm{Fe}^{3+}-$ to- $\mathrm{Fe}^{2+}$ transition is also suppressed by the incorporation of STO. The valence states of Fe ions are determined by X-ray photoelectron spectroscopy (XPS), as shown in Fig. $5 c$. The Fe $2 p_{3 / 2}$ peaks are fitted by LorentzianGaussian functions and the peaks corresponding to $\mathrm{Fe}^{2+}$ and $\mathrm{Fe}^{3+}$ are located at $709.9 \mathrm{eV}$ and $711.1 \mathrm{eV}$, respectively ${ }^{52}$. The percentage of $\mathrm{Fe}^{2+}$ is calculated according to the integration of the fitted peaks. The results (Fig. $5 \mathrm{~d}$ ) clearly demonstrate the decline of $\mathrm{Fe}^{2+}$ ratio from 31 to $12 \%$ as the STO content increases from 0.30 to 0.75 , indicating the significant stabilization of Fe valence and suppressed oxygen vacancies. The electric insulation of the BFSTO films is therefore improved, which further leads to the enhancements of breakdown strength and energy performance for the BFSTO films.

\section{Discussion}

We present a feasible domain engineering method that enables the development of new lead-free RFE materials and the realization of high-performance capacitive energy storage. It is revealed by HAADF-STEM that the long range FE order in BFO is interrupted by the incorporation of STO, resulting in the transformation of micron-scale FE domains into nano-scale PNRs. At the macroscopic level, the FE state with large hysteresis loss transits into a RFE state that maintains relatively high polarization but exhibits much suppressed hysteresis and remnant polarization. The favorable RFE property, together with the enhanced breakdown strengths, gives rise to giant energy storage densities of $\sim 70 \mathrm{~J} \mathrm{~cm}^{-3}$ in the BFSTO films with both $x=0.60$ and 0.75 , which are superior to other reported lead-free systems and rival the best lead-based systems. This corresponds with the 
first-principle simulations by $\mathrm{Xu}$ et al. ${ }^{55}$, proving the huge potential of BFO-based materials as high-energy-density dielectrics. It may be noted that the simulations predicted even higher energy density $\left(100-150 \mathrm{~J} \mathrm{~cm}^{-3}\right)$, indicating that there is still much room for further improvement in the BFSTO system.

The domain engineering technique proposed in our work is not only efficient in achieving targeted properties but also propagable in applications. Note that some other existing domain engineering methods, such as inducing anisotropy and stress in FE films by selected substrate ${ }^{56,57}$, or poling FE single crystals with selective directions to form a multi-domain configuration ${ }^{8}$, are actually limited by material categories and geometry parameters. As for the technique in this work, it can be readily applied in ceramics, single crystals as well as films. Moreover, it can be extended to the development of a number of other possible BFO-based RFEs such as $\mathrm{BFO}-\mathrm{CaTiO}, \mathrm{BFO}-\mathrm{BaZrO}_{3}$, and $\mathrm{BFO}-\mathrm{BaSnO}_{3}$ etc. We can also expect to obtain series of new RFE systems with domain engineering by incorporating the above analogous $\mathrm{ABO}_{3}$ components into other FEs such as $(\mathrm{K}, \mathrm{Na}) \mathrm{NbO}_{3}, \mathrm{LiNbO}_{3}$, and $\mathrm{LiTaO}_{3}$ etc. Considering that the mentioned systems have been seldom fabricated or investigated, we hope that the paradigm demonstrated in this contribution could motivate more efforts to shed light on new lead-free RFE materials and to develop promising candidates for dielectric energy storage.

\section{Methods}

Film fabrication. The BFSTO ceramic targets were sintered by a conventional solid state reaction method. To compensate the Bi evaporation during target sintering and film deposition, $10 \mathrm{~mol} \%$ excess Bi was added. The BFSTO films were fabricated using a PLD technique. A $\operatorname{KrF}$ laser excimer $(\lambda=248 \mathrm{~nm})$ with a repetition rate of $10 \mathrm{~Hz}$ was employed and the laser energy density was $\sim 1.7 \mathrm{~J} \mathrm{~cm}^{-2}$. The optimal deposition parameters were set to be $700^{\circ} \mathrm{C}$ with an oxygen pressure of 1.3 Pa. After deposition, the films were annealed at $500{ }^{\circ} \mathrm{C}$ with an oxygen pressure of $500 \mathrm{mbar}$ for $30 \mathrm{~min}$, and then cooled down to room temperature at a speed of $10^{\circ}$ $\mathrm{C} \mathrm{min}{ }^{-1}$. The thicknesses of the films are $\sim 500 \mathrm{~nm}$. Circular Au top electrodes (300 $\mu \mathrm{m}$ in diameter and $\sim 100 \mathrm{~nm}$ in thickness) were sputtered through a shadow mask to form the capacitor structures.

Characterization. The crystallinity and epitaxy properties of the BFSTO films were characterized using an X-ray diffractometer (XRD; Rigaku-2500). Surface and FE domain morphologies were revealed by an atomic force microscope (AFM, MFP 3D infinity, Oxford Instruments); and the cross-sectional image of the films were captured by a TEM, Philips CM200. The atomic interface morphology and domain structure images of the BFSTO films were obtain using a high-resolution scanning transmission electron microscope (STEM, JEOL ARM200CF). XPS measurements were conducted on a Thermo XPS ESCALAB 250Xi instrument (Thermo Scientific). Room-temperature dielectric permittivity and loss were measured with a precision impedance analyzer (HP 4294A, Agilent) with a perturbation voltage of $500 \mathrm{mV}$. Measurements of FE hysteresis loops, DC leakage current and FORC loops were performed on a ferroelectric test system (Precision Multiferroic II, Radiant Technologies, Inc.).

The FORC distribution. The distribution is derived from a series of FORC loops measurement. During the measurement, the FE film is exposed to a half-sinusoidal electric field that increases from the negative saturation field $-E_{\max }$ to $\alpha_{i}\left(-E_{\max }+\right.$ $i \Delta E)$ and then decreases to $\beta_{j}\left(-E_{\max }+j \Delta E ; j \leq i\right.$, Supplementary Fig. 8$)$, the measured polarization $P\left(\alpha_{i}, \beta_{j}\right)$ obeys the following equation ${ }^{32,33}$ :

$$
P\left(\alpha_{i}, \beta_{j}\right)-P\left(\alpha_{i}, \alpha_{i}\right)=-2 \int_{\beta_{j}}^{\alpha_{i}}\left(\int_{\beta_{j}}^{\alpha_{i}} p(\alpha, \beta) \mathrm{d} \alpha\right) \mathrm{d} \beta-2 \int_{\beta_{j}}^{\alpha_{i}} p_{\text {rev }}(\alpha) \mathrm{d} \alpha
$$

where $p(\alpha, \beta)$ is the irreversible distribution and $p_{\text {rev }}(\alpha)$ is the reversible distribution. Here, only the irreversible distribution is investigated because it gives the main information of FE hysteresis and nonlinearity. Differentiation of Eq. (3) with respect to $\alpha_{i}$ and $\beta_{j}$ gives

$$
\frac{\partial^{2} P\left(\alpha_{i}, \beta_{j}\right)}{\partial \alpha_{i} \partial \beta_{j}}=2 p\left(\alpha_{i}, \beta_{j}\right)
$$

In this work, $E_{\max }$ is set to be $1.5 \mathrm{MV} \mathrm{cm}^{-1}, \Delta \alpha=\Delta \beta=\Delta E=0.06 \mathrm{MV} \mathrm{cm}^{-1}$. An approximate method to calculate $p(\alpha, \beta)$ is

$$
p(\alpha, \beta) \approx \frac{1}{2} \frac{P(\alpha, \beta+\Delta \beta)-P(\alpha, \beta)-P(\alpha-\Delta \alpha, \beta+\Delta \beta)+P(\alpha-\Delta \alpha, \beta)}{\Delta \alpha \Delta \beta}
$$

In this way we get a discrete distribution $p(\alpha, \beta)$, which in fact represents the distribution of hysterons in the zone of $\alpha_{i}-\Delta \alpha \leq \alpha \leq \alpha_{i}$ and $\beta_{j}-\Delta \beta \leq \beta \leq \beta_{j}+\Delta \beta$ for $p(\alpha, \beta)=p\left(\alpha_{i}, \beta_{j}\right)(j \leq i)^{32}$.

Weibull distribution. A two-parameter Weibull distribution function of the electric breakdown strength is presented as

$$
P(E)=1-\exp \left(-\left(E / E_{\mathrm{b}}\right)^{\wedge} \gamma\right)
$$

where $P(E)$ is the cumulative probability of electric breakdown, $E$ is the measured breakdown field, $E_{\mathrm{b}}$ is the characteristic breakdown field corresponding to a $63.2 \%$ probability of failure and $\gamma$ is the Weibull modulus. For sample $1,2 \ldots n, E_{i}$ is the breakdown field of the $i$ th sample which is ranked in an increasing order. $P(E)_{i}=$ $i /(n+1)$ is numerically regarded as the cumulative probability of electric failure. Therefore, Eq. (6) transforms into $\log _{10}\left(-\ln \left(1-P(E)_{i}\right)\right) \sim \gamma \log _{10}\left(E_{i}\right)$, where $\gamma$ is the slope. In this work, 15 measurements of each composition were conducted for the Weibull fitting, i.e., $n=15$.

Data availability. All relevant data are available from the corresponding author upon reasonable request.

Received: 09 October 2017 Accepted: 10 April 2018

Published online: 08 May 2018

\section{References}

1. $\mathrm{Chu}, \mathrm{B}$. et al. A dielectric polymer with high electric energy density and fast discharge speed. Science 313, 334-336 (2006).

2. Cao, Y., Irwin, P. C. \& Younsi, K. The future of nanodielectrics in the electrical power industry. IEEE Trans. Dielectr. Electr. Insul. 11, 797-807 (2004).

3. Shen, Y., Zhang, X., Li, M., Lin, Y. \& Nan, C. Polymer nanocomposite dielectrics for electrical energy storage. Natl. Sci. Rev. 4, 23-25 (2017).

4. Chen, Q., Shen, Y., Zhang, S. \& Zhang, Q. M. Polymer-based dielectrics with high energy storage density. Annu. Rev. Mater. Res. 45, 433-458 (2015).

5. Yao, Z. et al. Homogeneous/inhomogeneous-structured dielectrics and their energy-storage performances. Adv. Mater. 29, 1601727 (2017).

6. $\mathrm{Li}, \mathrm{Q}$. et al. Flexible high-temperature dielectric materials from polymer nanocomposites. Nature 523, 576-579 (2015).

7. Zhang, X. et al. Giant energy density and improved discharge efficiency of solution-processed polymer nanocomposites for dielectric energy storage. $A d v$. Mater. 28, 2055-2061 (2016).

8. Sun, E. \& Cao, W. Relaxor-based ferroelectric single crystals: growth, domain engineering, characterization and applications. Prog. Mater. Sci. 65, 124-210 (2014).

9. Yang, Z. et al. Significantly enhanced recoverable energy storage density in potassium-sodium niobate-based lead free ceramics. J. Mater. Chem. A $\mathbf{4}$, 13778-13785 (2016).

10. Xie, Z., Peng, B., Meng, S., Zhou, Y. \& Yue, Z. High-energy-storage density capacitors of $\mathrm{Bi}\left(\mathrm{Ni}_{1 / 2} \mathrm{Ti}_{1 / 2}\right) \mathrm{O}_{3}-\mathrm{PbTiO}_{3}$ thin films with good temperature stability. J. Am. Ceram. Soc. 96, 2061-2064 (2013).

11. Kwon, D. \& Lee, M. H. Temperature-stable high-energy-density capacitors using complex Perovskite thin films. IEEE T. Ultrason. Ferr. 59, 1894-1899 (2012).

12. Sun, Z. et al. Ultrahigh energy storage performance of lead-free oxide multilayer film capacitors via interface engineering. Adv. Mater. 29, 1604427 (2017).

13. Ederer, C. \& Spaldin, N. A. Effect of epitaxial strain on the spontaneous polarization of thin film ferroelectrics. Phys. Rev. Lett. 95, 257601 (2005).

14. Shen, Z., Wang, X., Luo, B. \& Li, L. $\mathrm{BaTiO}_{3}-\mathrm{BiYbO}_{3}$ perovskite materials for energy storage applications. J. Mater. Chem. A 3, 18146-18153 (2015).

15. Triamnak, N., Yimnirun, R., Pokorny, J. \& Cann, D. P. Relaxor characteristics of the phase transformation in $(1-\mathrm{x}) \mathrm{BaTiO}_{3}-\mathrm{xBi}\left(\mathrm{Zn}_{1 / 2} \mathrm{Ti}_{1 / 2}\right) \mathrm{O}_{3}$ Perovskite ceramics. J. Am. Ceram. Soc. 96, 3176-3182 (2013).

16. Rojac, T. et al. $\mathrm{BiFeO} 3$ ceramics: processing, electrical, and electromechanical properties. J. Am. Ceram. Soc. 97, 1993-2011 (2014).

17. Wei, Y., Wang, X., Zhu, J., Wang, X. \& Jia, J. Dielectric, ferroelectric, and piezoelectric properties of $\mathrm{BiFeO}_{3}-\mathrm{BaTiO}_{3}$ ceramics. J. Am. Ceram. Soc. 96, 3163-3168 (2013)

18. Mishra, A., Majumdar, B. \& Ranjan, R. A complex lead-free $(\mathrm{Na}, \mathrm{Bi}, \mathrm{Ba})(\mathrm{Ti}, \mathrm{Fe})$ $\mathrm{O}_{3}$ single phase perovskite ceramic with a high energy-density and high 
discharge-efficiency for solid state capacitor applications. J. Eur. Ceram. Soc. 37, 2379-2384 (2017).

19. Sharma, S., Singh, V. \& Dwivedi, R. K. Electrical properties of $(1-\mathrm{x}) \mathrm{BFO}-(\mathrm{x})$ PZT multiferroics synthesized by sol-gel method: transition from relaxor to non-relaxor. J. Alloy. Compd. 682, 723-729 (2016).

20. Correia, T. M. et al. A lead-free and high-energy density ceramic for energy storage applications. J. Am. Ceram. Soc. 96, 2699-2702 (2013).

21. Pan, $\mathrm{H}$. et al. $\mathrm{BiFeO}_{3}-\mathrm{SrTiO}_{3}$ thin film as a new lead-free relaxor-ferroelectric capacitor with ultrahigh energy storage performance. J. Mater. Chem. A $\mathbf{5}$, 5920-5926 (2017).

22. Saad, Y. et al. Dielectric response of ceramic $\mathrm{Sr}_{2-\mathrm{x}} \mathrm{Bi}_{\mathrm{x}} \mathrm{Ti}_{2-\mathrm{x}} \mathrm{Fe}_{\mathrm{x}} \mathrm{O}_{6}(0 \leq \mathrm{x} \leq 1.5)$ perovskites. J. Phys. Chem. Solids 81, 40-49 (2015).

23. Tong, S. et al. Lead Lanthanum Zirconate Titanate ceramic thin films for energy storage. ACS Appl. Mater. Interfaces 5, 1474-1480 (2013).

24. Sun, Z. et al. Large energy density, excellent thermal stability and high cycling endurance of lead-free $\mathrm{BaZr}_{0.2} \mathrm{Ti}_{0.8} \mathrm{O}_{3}$ film capacitors. ACS Appl. Mater. Interfaces 9, 17096 (2017).

25. $\mathrm{Hu}, \mathrm{W}$. et al. Electron-pinned defect-dipoles for high-performance colossal permittivity materials. Nat. Mater. 12, 821-826 (2013).

26. Correia, T., Stewart, M., Ellmore, A. \& Albertsen, K. Lead-free ceramics with high energy density and reduced losses for high temperature applications. Adv. Eng. Mater. 19, 1700019 (2017).

27. Tang, X., Zhu, X., Dai, J. \& Sun, Y. Self-limited grain growth, dielectric, leakage and ferroelectric properties of nanocrystalline $\mathrm{BiFeO}_{3}$ thin films by chemical solution deposition. Acta Mater. 61, 1739-1747 (2013).

28. Peng, B., Xie, Z., Yue, Z. \& Li, L. Improvement of the recoverable energy storage density and efficiency by utilizing the linear dielectric response in ferroelectric capacitors. Appl. Phys. Lett. 105, 52904 (2014)

29. Xie, $\mathrm{Z}$. et al. Highly (100)-oriented $\mathrm{Bi}\left(\mathrm{Ni}_{1 / 2} \mathrm{Hf}_{1 / 2}\right) \mathrm{O}_{3}-\mathrm{PbTiO}_{3}$ relaxorferroelectric films for integrated piezoelectric energy harvesting and storage system. J. Am. Ceram. Soc. 98, 2968-2971 (2015).

30. Hao, X., Wang, Y., Zhang, L., Zhang, L. \& An, S. Composition-dependent dielectric and energy-storage properties of $(\mathrm{Pb}, \mathrm{La})(\mathrm{Zr}, \mathrm{Sn}, \mathrm{Ti}) \mathrm{O}_{3}$ antiferroelectric thick films. Appl. Phys. Lett. 102, 163903 (2013).

31. Zhang, Y. et al. Mn doping to enhance energy storage performance of leadfree 0.7 NBT- 0.3 ST thin films with weak oxygen vacancies. Appl. Phys. Lett. 110, 243901 (2017).

32. Hu, Z., Ma, B., Koritala, R. E. \& Balachandran, U. Temperature-dependent energy storage properties of antiferroelectric $\mathrm{Pb}_{0.96} \mathrm{La}_{0.04} \mathrm{Zr}_{0.98} \mathrm{Ti}_{0.02} \mathrm{O}_{3}$ thin films. Appl. Phys. Lett. 104, 263902 (2014).

33. Park, M. H. et al. Thin $\mathrm{Hf}_{\mathrm{x}} \mathrm{Zr}_{1-\mathrm{x}} \mathrm{O}_{2}$ films: a new lead-free system for electrostatic supercapacitors with large energy storage density and robust thermal stability. Adv. Energy Mater. 4, 1400610 (2014).

34. Yang, S. M., Kim, T. H., Yoon, J. \& Noh, T. W. Nanoscale observation of timedependent domain wall pinning as the origin of polarization fatigue. $A d v$. Funct. Mater. 22, 2310-2317 (2012).

35. Zheng, D., Zuo, R., Zhang, D. \& Li, Y. Novel $\mathrm{BiFeO}_{3}-\mathrm{BaTiO}_{3}-\mathrm{Ba}\left(\mathrm{Mg}_{1 / 3} \mathrm{Nb}_{2 / 3}\right)$ $\mathrm{O}_{3}$ lead-free relaxor ferroelectric ceramics for energy-storage capacitors. J. Am. Ceram. Soc. 98, 2692-2695 (2015).

36. Khanchaitit, P., Han, K., Gadinski, M. R., Li, Q. \& Wang, Q. Ferroelectric polymer networks with high energy density and improved discharged efficiency for dielectric energy storage. Nat. Commun. 4, 2845 (2013).

37. Fujii, I. Dielectric nonlinearity of ferroelectrics. BSc dissertation, Pennsylvania State Univ. (2010).

38. Piazza, D., Stoleriu, L., Mitoseriu, L., Stancu, A. \& Galassi, C. Characterisation of porous PZT ceramics by first-order reversal curves (FORC) diagrams. J. Eur. Ceram. Soc. 26, 2959-2962 (2006).

39. Fujii, I., Trolier-McKinstry, S. \& Nies, C. Effect of grain size on dielectric nonlinearity in model $\mathrm{BaTiO}_{3}$-based multilayer ceramic capacitors. J. Am. Ceram. Soc. 94, 194-199 (2011).

40. Wu, L., Wang, X., Shen, Z., Li, L. \& Feteira, A. Ferroelectric to relaxor transition in $\mathrm{BaTiO}_{3}-\mathrm{Bi}\left(\mathrm{Zn}_{2 / 3} \mathrm{Nb}_{1 / 3}\right) \mathrm{O}_{3}$ ceramics. J. Am. Ceram. Soc. 100, 265-275 (2017).

41. Deluca, M. et al. High-field dielectric properties and Raman spectroscopic investigation of the ferroelectric-to-relaxor crossover in $\mathrm{BaSn}_{\mathrm{x}} \mathrm{Ti}_{1-\mathrm{x}} \mathrm{O}_{3}$ ceramics. J. Appl. Phys. 111, 84102 (2012).

42. Shvartsman, V. V. \& Lupascu, D. C. Lead-free relaxor ferroelectrics. J. Am. Ceram. Soc. 95, 1-26 (2012).

43. Akbarzadeh, A. R., Prosandeev, S., Walter, E. J., Al-Barakaty, A. \& Bellaiche, L. Finite-temperature properties of $\mathrm{Ba}(\mathrm{Zr}, \mathrm{Ti}) \mathrm{O}_{3}$ relaxors from first principles. Phys. Rev. Lett. 108, 257601 (2012).

44. Prosandeev, S., Wang, D., Akbarzadeh, A. R., Dkhil, B. \& Bellaiche, L. Fieldinduced percolation of polar nanoregions in relaxor ferroelectrics. Phys. Rev. Lett. 110, 20760120 (2013).

45. Bokov, A. A. \& Ye, Z. G. Recent progress in relaxor ferroelectrics with perovskite structure. J. Mater. Sci. 41, 31-52 (2006).
46. Tang, Y. L. et al. Observation of a periodic array of flux-closure quadrants in strained ferroelectric $\mathrm{PbTiO}_{3}$ films. Science 348, 547-551 (2015).

47. Ogihara, H., Randall, C. A. \& Trolier-McKinstry, S. Weakly coupled relaxor behavior of $\mathrm{BaTiO}_{3}-\mathrm{BiScO}_{3}$ ceramics. J. Am. Ceram. Soc. 92, 110-118 (2009).

48. Dittmer, R., Jo, W., Rödel, J., Kalinin, S. \& Balke, N. Nanoscale insight into lead-free BNT-BT-xKNN. Adv. Funct. Mater. 22, 4208-4215 (2012).

49. Ishiwara, $\mathrm{H}$. Impurity substitution effects in $\mathrm{BiFeO}_{3}$ thin films-From a viewpoint of FeRAM applications. Curr. Appl. Phys. 12, 603-611 (2012).

50. Chiu, F. A review on conduction mechanisms in dielectric films. Adv. Mater. Sci. Eng. 2014, 1-18 (2014).

51. Pabst, G. W., Martin, L. W., Chu, Y. \& Ramesh, R. Leakage mechanisms in $\mathrm{BiFeO}_{3}$ thin films. Appl. Phys. Lett. 90, 72902 (2007).

52. Wang, Y. \& Nan, C. Enhanced ferroelectricity in Ti-doped multiferroic $\mathrm{BiFeO}_{3}$ thin films. Appl. Phys. Lett. 89, 52903 (2006).

53. Guo, Y. et al. Critical roles of $\mathrm{Mn}$-ions in enhancing the insulation, piezoelectricity and multiferroicity of $\mathrm{BiFeO}_{3}$-based lead-free high temperature ceramics. J. Mater. Chem. C. 3, 5811-5824 (2015).

54. Khan, M. A., Comyn, T. P. \& Bell, A. J. Leakage mechanisms in bismuth ferrite-lead titanate thin films on Pt/Si substrates. Appl. Phys. Lett. 92, 72908 (2008).

55. Xu, B., Îniguez, J. \& Bellaiche, L. Designing lead-free antiferroelectrics for energy storage. Nat. Commun. 8, 15682 (2017).

56. Jang, H. W. et al. Domain engineering for enhanced ferroelectric properties of epitaxial (001) BiFeO thin films. Adv. Mater. 21, 817-823 (2009).

57. Shelke, V. et al. Reduced coercive field in $\mathrm{BiFeO}_{3}$ thin films through domain engineering. Adv. Mater. 23, 669-672 (2011).

\section{Acknowledgements}

This work was supported by Basic Science Center Project of NSFC under grant No. 51788104, and the Natural Science Foundation of China (Grant Nos. 51532003 and 51729201). Q.Z. and L.G. thank the National Program on Key Basic Research Project (Grant No. 2014CB921002), the Strategic Priority Research Program of Chinese Academy of Sciences (Grant No. XDB07030200) and the Natural Science Foundation of China (Grant Nos. 51522212, 51421002, and 51672307). We thank Dr. Deyang Chen (South China Normal University) for helpful discussions on the AFM tests.

\section{Author contributions}

H.P. and Y.-H.L. conceived and performed the study. H.P. and Y.-J.Z. fabricated the films. Jing M. and Ji M. conducted the AFM test. Q.Z., X.L., B.G., and L.G. performed the TEM and STEM measurements. H.P., X.Z., Y.S., and C.-W.N. conducted the dielectric and ferroelectric measurements. H.P., Ji M., and L.L. performed the XRD and XPS measurements. H.P. and Y.-H.L. wrote the shaped manuscript. All authors discussed the results and commented on the manuscript.

\section{Additional information}

Supplementary Information accompanies this paper at https://doi.org/10.1038/s41467018-04189-6.

Competing interests: The authors declare no competing interests.

Reprints and permission information is available online at http://npg.nature.com/ reprintsandpermissions/

Publisher's note: Springer Nature remains neutral with regard to jurisdictional claims in published maps and institutional affiliations.

Open Access This article is licensed under a Creative Commons Attribution 4.0 International License, which permits use, sharing, adaptation, distribution and reproduction in any medium or format, as long as you give appropriate credit to the original author(s) and the source, provide a link to the Creative Commons license, and indicate if changes were made. The images or other third party material in this article are included in the article's Creative Commons license, unless indicated otherwise in a credit line to the material. If material is not included in the article's Creative Commons license and your intended use is not permitted by statutory regulation or exceeds the permitted use, you will need to obtain permission directly from the copyright holder. To view a copy of this license, visit http://creativecommons.org/ licenses/by/4.0/.

(C) The Author(s) 2018 